\title{
Online Hazard Maps, Risk Communication, Acceptance and Usage Continuance Intention Model: A Selected Review of Literature
}

\author{
Kartika Puspita Sari ${ }^{a}$ *, Hidehiko Kanegae ${ }^{\mathrm{b}}$ \\ a Graduate School of Policy Science - Ritsumeikan University, ps0204fr@ed.ritsumei.ac.jp \\ ${ }^{b}$ Graduate School of Policy Science - Ritsumeikan University, hkanegae@sps.ritsumei.ac.jp \\ * Corresponding author
}

Keywords: Online Hazard Maps, Risk Communication, Protective Action Decision Model, Technology Acceptance Model, Expectation-Confirmation Theory

\begin{abstract}
:
The rapid progress of communication and information technology, as well as geospatial technology in the last decades, has noticeably altered the manner in which spatial information about disasters is accessed, stored and disseminated. The Internet broadcasts static maps and interactive mapping about natural hazards, in which as a consequence, the public is now flooded by vast amounts of this cartographic information. However, there are a little theoretical or empirical study concerns how individuals accept online hazard maps as a source of information or a medium of risk communication. As an initial step of further research about the issue, this study, therefore, aims to conduct a systematic review on relevant prior research that can help to construct a model to define how individuals accept and intend to continue using this webbased cartographic communication medium. As a next step, we will extend this model to explain how the acceptance and intention might increase one's awareness of the risks of natural hazards as it subsequently affects decision making on risk reduction behavioral responses. We begin the analysis by reviewing the Protective Action Decision Model (PADM) as the foundational framework for risk communication. As acceptance and usage intention is commonly described by Technology Acceptance Model (TAM) and Expectation-Confirmation Theory (ECT), we then comprehensively review selected literature on these notions. Various studies conducted within hazard maps, the Internet and risk communication context were evaluated to identify factors have not been incorporated into prior acceptance and usage models. The result of this study contributes to the theoretical novelty and helps to identify gaps of existing literature in the domains of user acceptance and intention of continue usage of online hazard maps in risk communication.
\end{abstract}

